\title{
GEOMETRICALLY SIMILAR RECTANGULAR PASSIVE MICROMIXERS AND THE SCALING VALIDITY ON MIXING EFFICIENCY AND PRESSURE DROPS
}

\author{
NARESH Veldurthi' ${ }^{1}$, BODAS D. ${ }^{2}$, SUNIL Chandel ${ }^{3}$, TEJASHREE Bhave ${ }^{4}$ \\ ${ }^{I}$ Department of Applied Physics, Defence Institute of Advanced Technology, \\ Girinagar, Pune-411025, India, e - mail: veldurthi.naresh@gmail.com \\ ${ }^{2}$ Centre for Nanobioscience, Agharkar Research Institute, Pune-411004, \\ India.e - mail:dsbodas@aripune.org \\ ${ }^{3}$ Department of Mechanical Engineering, Defence Institute of Advanced Technology, \\ Girinagar, Pune-411025, India, e - mail: suniliitd2003@gmail.com \\ ${ }^{4}$ Department of Applied Physics, Defence Institute of Advanced Technology, \\ Girinagar, Pune-411025, India, e - mail: tejab68@gmail.com
}

\begin{abstract}
In the present work, two geometrically similar passive geometries with dumbbell shape were designed to perturb the dominating viscous forces in the low Reynolds number $(\mathrm{Re})$ flows of the fluids. The geometries were designated as PDM-I and PDM-II, in which all the linear dimensions were related by a constant scale factor of two. Mixing efficiencies and pressure drops of the species at various Reynolds number (Re) were calculated to estimate the scaling effect validations. Finally, the geometrically similar PDM geometries were fabricated in Polydimethylsiloxane (PDMS) polymer to evaluate the scaling effect on the mixing efficiencies of the dyes and validated with the simulation results of species mixing.
\end{abstract}

KEYWORDS: Passive micromixer, CFD, rectangular dumbbell, Polydimethylsiloxane (PDMS).

\section{Introduction}

Microfluidics has taken inspiration from various fields such as microelectronics, microanalysis and bio-defence. It is very useful in microbiology since it requires very small volume of sample and reagents. It is very useful in analytical microbiology and microanalysis. Microfluidics based applications facilitate to accommodate several functions in a small and cost effective device which has led to popularity of microfluidic device. It also has been a focus of research in military technologies for the purpose of on site, fast and in situ detection of potential bacteriological threats and pollutions. Many government and military establishment are therefore interested in the development of novel microfluidic devices.

Ever since the inception of the microfluidics research, micro devices with simple and integrated microchannels, chambers, valves and pumps are being engineered to cater the complex tasks in the analytical [1], biological [2] and chemical processes [3]. The controlled chemistry [4] and molecular manipulation [5] in the micro devices, makes them very much desirable for such applications. Moreover, the physics of these devices is very much intriguing during the fluid dynamics inside the constricted structures. The design and fabrication of micro/nano devices were explored tremendously with the advent of economically feasible materials [6] and tangible technologies [7-9]. The application-oriented research of microfluidics propelled the researchers to develop [10] very complex micro and nanoscale devices. In other words, the reign of lab-on-a-chip (LOC) devices and micro-total analytical systems ( $\mu$-TAS) has evolved.

Micromixers are vital and are integral part of $\mu$-TAS and LOC devices for initial premixing of bio and chemical samples. The chemical and biological processes often involve 
mixing of species or reactants to initiate the process. Chemical processes [3] (such as emulsification, foaming and blending), as well as biological processes [2], (such as drug delivery, protein folding and cell activation) require initial mixing for initiation. In the microsystems, the passive micromixers perform the physical mixing of fluids by increasing the area of contact for diffusion of fluids by split-recombine [11, 12], creation of recirculation zones [13, 14], stretching-folding [15] through geometrical configuration and complex flow pattern. On the other hand, there are active micromixers [16, 17], which do mixing of fluids by external energy like electro kinetics, magnetic, dielectrophoretics, acoustics on the microgeometries that agitate and mix the fluids efficiently than passive micromixers.

In the mechanism of mixing in the passive micromixers, the dimensions and the geometrical configuration of the microdevices induce diffusion and chaotic advection. In the case of active micromixers, the oscillatory and the rotational motion of the piezo, acoustic, magnetic based actuators in the microdevices induce turbulence motion of the fluid streamlines. Pertaining to the comprehension of mixing in the passive micromixers, the early pioneering work [18] determined the transport phenomenon in the simple T-shaped micromixer. In this, the inter-diffusion of analyte and the device characteristics were studied experimentally and analytically. In other work [19], scaling effects on the transverse diffusion near the surface of the channel were studied again in a simple Y-shaped micromixer. Following these, the molecular transverse diffusion have been analysed experimentally and theoretically, and the similar scaling laws have been deduced [20, 21]. Subsequently, several numerical analyses were carried out to comprehend the diffusion transport and cross-stream diffusion in the rectangular microchannels [22]. Microfluidics research, micro devices with simple and integrated microchannels, chambers, valves and pumps

However, the scaling effect on the chemical species mixing was not much explored in the passive micromixers. Scaling is important in upscaling of new technologies like heat exchangers and internal combustion engines [27, 28]. In the current work, two geometrically similar rectangular passive micromixers designated as PDM-I and PDM-II were fabricated in Polydimethylsiloxane (PDMS) by conventional softlithography methods. Since the PDM structures are geometrically similar, the dimensions of the PDM-II geometry differ by a constant scale factor of two with PDM-I. Bi and Se species have been chosen, which do undergo diffusion and advection in the rectangular PDM geometries and the resulting complexes were characterized and analyzed with respect to scaling effect. In the comprehension of scaling effect deviation on the $\mathrm{Bi}_{2} \mathrm{Se}_{3}$ complexes resulted from the PDM geometries, modeling and simulations of the PDM-I and II geometries were performed in ANSYS FLUENT. Despite the dimensions of the geometries differ by a constant scale factor of two, the mixing efficiencies of the water species revealed from the simulations didn't show any scaling relation. While the pressure drops of the species flow at different Reynolds number (Re) holds. This work explores the dimensional factors that deter the scaling effect in the geometrically similar passive micromixers by modeling and simulation of other two rectangular passive dumbbell micromixer geometries (PDM-III and PDM-IV) which vary linearly only in one dimension to that of PDM-I. Further, in the simulations the transverse and longitudinal diffusion of species at top and bottom walls and at centre of the outlet of the PDM geometries have been discussed, in view of the deviation from the scaling effect. In addition to the simulations of the PDM geometries, dimensional analysis was invoked to relate the parameters of interest in the geometrically similar structures. Herein the work, mixing efficiency and pressure drop parameters of the PDM I and II geometries were related theoretically, considering the dependent factors of the parameters. 

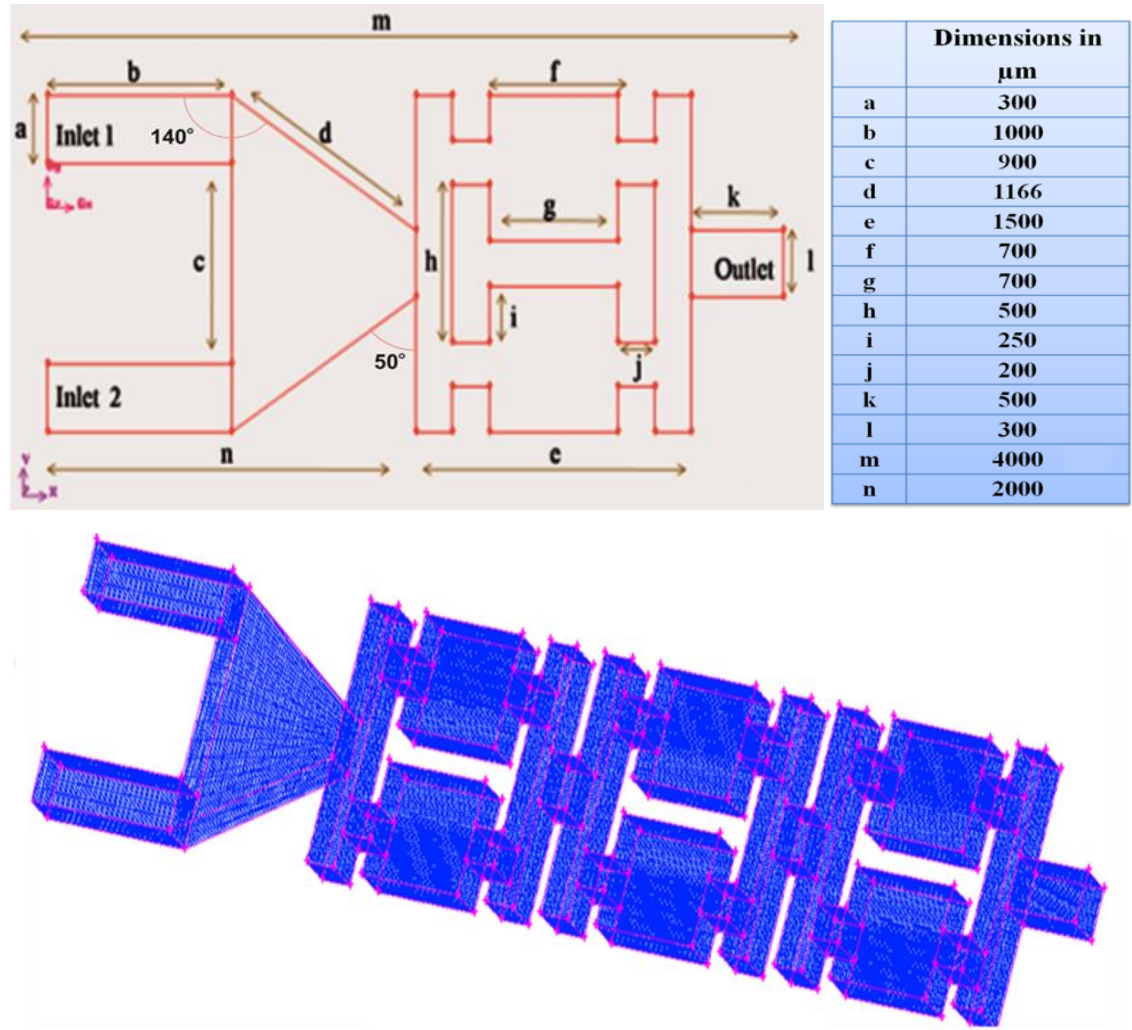

Fig. 1 Schematic of theGeometry of the Passive dumbbell micromixer (PDM), model (b) Structured mesh of the PDM-II geometry with hexahedral cells.

\section{Design and Geometry of the Rectangular Passive Micromixers}

Passive micromixer geometries were designed with rectangular dumbbell shape as the obstruction, since that the passive micromixers are termed as passive dumbbell micromixers (PDM). The number of rectangular dumbbell units was increased axially with constant spacing in each of the geometry to enhance the diffusion of species in the micromixer. The schematic of the PDM-II geometry is depicted in Fig 1(a). The rectangular dumbbell shaped obstructions are $900 \times 500 \mu \mathrm{m}(\mathrm{L} \times \mathrm{W})$ placed at center inside the mixing area of the PDM designs as shown in the Fig 1(a). The rectangular dumbbell shape was selected as the obstruction, since it splits the species streamlines sharply due to their $90^{\circ}$ turns inside the design. Also, the geometries have square notches above and below the rectangular dumbbell as shown in Fig 1(a). The PDM I geometry with five dumbbell units comprises the length of $(10.8 \mathrm{~mm})$ along $\mathrm{X}$ which is the flow direction, width $(1.5 \mathrm{~mm})$ along $\mathrm{y}$-direction and height $(150 \mu \mathrm{m})$ along z-direction. The PDM-II geometry dimensions are exactly double to that of the PDM-I, while the PDM-III and PDM-IV have dimensions double to that of the PDM- I only along $\mathrm{Y}$ and $\mathrm{Z}$ directions respectively. The repetitive dumbbell units in all the geometries were varied from 1 to 5 .

\subsection{Modeling and Simulations of PDM Geometries}

The PDM geometries were modeled by using the Gambit software and the simulations of the flow under consideration were performed by means of commercial code FLUENT. Threedimensional numerical analysis was performed for the PDM geometries by assuming the steady state, incompressible and laminar flow conditions. The governing fluid flow equations in the simulations are continuity, momentum and convection-diffusion equations given in Eq's (1), (2) \& (3) respectively. 


$$
\begin{gathered}
\frac{\partial u}{\partial x}+\frac{\partial v}{\partial y}+\frac{\partial w}{\partial z}= \\
\rho u \frac{\partial u}{\partial x}+\rho v \frac{\partial v}{\partial y}+\rho w \frac{\partial w}{\partial z}=-\frac{\partial p}{\partial x}+\mu\left(\frac{\partial^{2} u}{\partial x^{2}}+\frac{\partial^{2} v}{\partial y^{2}}+\frac{\partial^{2} w}{\partial z^{2}}\right) \\
\left(\vec{u} \cdot\left(\frac{\partial u}{\partial \mathrm{x}}+\frac{\partial v}{\partial \mathrm{y}}+\frac{\partial w}{\partial \mathrm{z}}\right) C\right)=D\left(\frac{\partial^{2} u}{\partial x^{2}}+\frac{\partial^{2} v}{\partial y^{2}}+\frac{\partial^{2} w}{\partial z^{2}}\right) C
\end{gathered}
$$

Where $\rho$ is the density of the fluid, $u, v \& w$ are the velocity vectors, $\mu$ is the kinematic viscosity of the fluid, $C$ is the concentration of the species and $D$ is the mass diffusivity of the species. Here the temperature of fluid (water) is $27^{\circ} \mathrm{C}$. The viscosity and density of fluid is 0.0008502 Pa.s and $996.51 \mathrm{~kg} / \mathrm{m}^{3}$.

Water species were considered for the fluid flow simulations in the all of the PDM geometries. Water was flown through inlet 1 and inlet 2 with the mass diffusivity value of water taken as $10^{-9} \mathrm{~m}^{2} / \mathrm{sec}$, implemented by the constant-dilute approximation method. Velocity inlet, outflow and no-slip boundary conditions were chosen for inlets, outlet and walls of the passive micromixer geometries respectively. In order to achieve the desired accuracy of the calculations the equations were discretized using second order scheme for pressure and the momentum, water species and energy were discretized with the second order up winding method. The pressure-velocity coupling in the segregated solver was applied with the SIMPLE method. The residuals of the governing equations or the convergence monitor of the equations was set at $10^{-5}$. Grid generation for the geometries was done in GAMBIT. The geometries were discretized with hexahedral cells, thus making the geometry a structured mesh over which the governing equations Eq's (1), (2) \& (3) can be solved by the SIMPLE algorithm. For the present numerical investigations, hexahedral cells were chosen because it was found from literatures that for the same cell amount, the accuracy of solutions in hexahedral meshes is the highest.

Mesh independence tests of the geometries were performed in terms of the pressure drop. The calculations were performed for the single rectangular dumbbell unit of the PDM-III geometry with 22540, 76236 and 177600 hexahedral cells and the difference of pressure drop found between the last two cells number was less than 5\%. Hence, 76,236 cells were chosen for further parametric investigation. The edge meshing used for the geometry was, for $0.4 \mathrm{~mm}$ of length 15 nodes were used and this was repeated further for all the five rectangular dumbbell units of the PDM-III geometry. The same criterion was applied for the PDM-II geometry, where the inlet channel width was $600 \mu \mathrm{m}$. In addition, mesh independency tests were performed for the PDM-I geometry, where the inlet channel width was $300 \mu \mathrm{m}$. For the single rectangular dumbbell unit, the test was done with 11524, 39132 and 89096 hexahedral cells. Beyond 39132 cells, the solution varied by less than 3\%. Therefore, for further calculations 39132 cells were used. In this, for $0.2 \mathrm{~mm}$ edge length of the geometry, meshing was done with 11 nodes. This value was repeated for all the five rectangular dumbbell units of PDM-I and PDM-IV geometries. The simulated geometries were meshed with only the hexahedral cells to minimize the numerical diffusion and the uniformity maintained among the equal width dimension geometries to avoid the discrepancies in the solutions. The meshed PDM-II geometry with four rectangular dumbbell units is represented in Fig 1(b), the inset shows the exploded view of the hexahedral meshed mixing unit. The simulations of the species flown in the PDM geometries were done in the low Reynolds no (Re) regime (0.02 to 0.20). The width and height dimensions of all the modelled and simulated PDM geometries are given in Table I. The mixing efficiency of the species were calculated by the Eq. (4) given as [24].

$$
\operatorname{Mixingefficiency}(\eta)=\left(1-\sqrt{\left(\frac{\sigma^{2}}{\sigma_{\max }^{2}}\right)}\right) \times 100 \%
$$




$$
\sigma^{2}=\frac{1}{N} \sum_{i=1}^{N}\left(C_{i}-\bar{C}_{m}\right)^{2}
$$

where $\mathrm{N}$ is the no. of data points on the outlet plane, $C_{i}$ is the mass fraction of the species in the outlet at data point $i$ and $\bar{C}_{m}$ is the average mass fraction of the data points at the outlet. $\sigma_{\max }^{2}$ is the maximum variance of the species concentration taken as 0.5 .

Table. 1: Inlet channel width and height of the simulated PDM geometries

\begin{tabular}{|c|c|c|}
\hline Geometry & Inlet channel Width & Channel Height \\
\hline PDM-I & $300 \mu \mathrm{m}$ & $150 \mu \mathrm{m}$ \\
\hline PDM-II & $600 \mu \mathrm{m}$ & $300 \mu \mathrm{m}$ \\
\hline PDM-III & $600 \mu \mathrm{m}$ & $150 \mu \mathrm{m}$ \\
\hline PDM-IV & $300 \mu \mathrm{m}$ & $300 \mu \mathrm{m}$ \\
\hline
\end{tabular}

\section{Results and Discussion}

In the numerical solving, the water species mixing and the related pressure drops were analysed at low Re flows of the species for one to five similar mixing units of both the geometries. Fig 2 represents the mixing efficiency of the water species and the pressure drops calculated at different $\operatorname{Re}(0.02$ to 0.2$)$ for the PDM-I and II geometries. The mixing of the species in the microscale passive geometries is dependent solely on the diffusion and on the

geometrical configuration or design. The rectangular dumbbell like structures efficiently perturb the laminar species flow and the sequential similar units up to five numbers render maximum interfacial area of the species by splitting and recombining. From the Figs 2(a) and 2(c), the mixing efficiency of the species is highest.

For the PDM geometry with five rectangular dumbbell units. PDM-I attains the $93 \%$ mixing efficiency of species at $\operatorname{Re}=0.02(100 \mu \mathrm{m} / \mathrm{sec})$ as shown in Fig 2(a), while, the PDMII with the same number of units achieves $79 \%$ (Fig 2(c)) mixing efficiency at $\operatorname{Re}=0.04$ (100 $\mu \mathrm{m} / \mathrm{sec})$. The mixing efficiencies of the species decrease with increase in Re for both the geometries irrespective of the dumbbell units, since the diffusion time of the species are reduced or affect the residence time of the species. In the geometrically similar PDM-I and PDM-II, the mixing efficiencies of PDM-I are higher for every rectangular dumbbell units to that of PDM-II, as shown in Fig 2(a) and 2(c) respectively. In view of scaling effect, the mixing efficiencies of the species in PDM-II are to be half to that of PDM-I mixing efficiencies, but this not evident from the fig 2(c) and 2(a) respectively. In the case of pressure drops, PDM-I exhibits higher at every flow velocity of species to that of the PDM-II and for every rectangular dumbbell units as shown in Fig 2(b) and 2(d) respectively. The values are in agreement with the scaling effect, where the pressure drop values of PDM-II are half to that of PDM-I. In this regard, the modelling and simulations of PDM-III and IV passive micromixers are significant, which may explain the geometrical factors that influence mixing efficiencies of species and pressure drops in PDM-I and II geometries.

In the Fig 3(a) and 3(b), the mixing efficiencies and pressure drops of the PDM-III geometry with only width of the geometry $(600 \mu \mathrm{m})$ increased to that of PDM-I geometry is shown. The geometry was simulated with the same flow conditions as that of other PDM geometries. The mixing efficiencies of species in PDM-III decreased to that of PDM-I geometry, but were same to that of PDM-II with 0 to $5 \%$ variation (for 1 to 5 dumbbell units). In the case of pressure drops of PDM-III, the values shown in Fig 3(b) were seen double to that of the PDM-II geometry, but were same to that of PDM-I pressure drops. Due to increase in width of the PDM-III, the velocities of the species streamlines will be same except near and at the walls of the geometry along transverse direction i.e., non-uniform 
velocities across the geometry will reduce. This makes the cross-stream diffusion or transverse diffusion drops to minimum at centre and relatively more diffusion of species at the top and bottom walls of the channel. Therefore, the mixing efficiencies decrease when the width of the geometry is increased. Next, PDM-IV geometry (aspect ratio $=1$ ) was simulated in which only the height of the geometry $(300 \mu \mathrm{m})$ was doubled, while the width was same to that of PDM-I geometry. Fig 3(c) and 3(d) depict the mixing efficiency and pressure drops of the PDM-IV geometry respectively. The mixing efficiencies of the PDM-IV are same as that of PDM-I, but increased to that of PDM-II at the respective flow velocities of the species. In the case of pressure drops of PDM-IV (Fig 3(d)), which remained same as that of PDM-II, but were half to that of PDM-I. Since the increase in height of the PDM-IV geometry induces uniform velocities along depth wise, except near and at the walls which in turn reduce magnitude of the wall shear stress $\left(\tau_{w}\right)$. Hence, there will be less drop in pressure values, which occur as per Eq. (6).

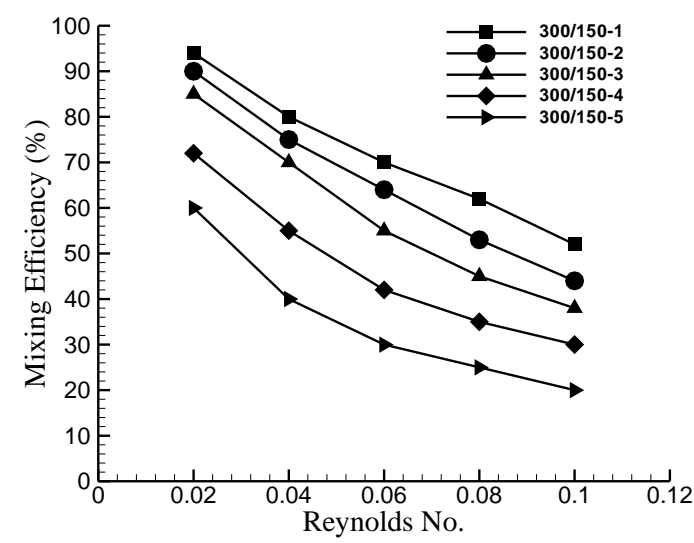

(a)

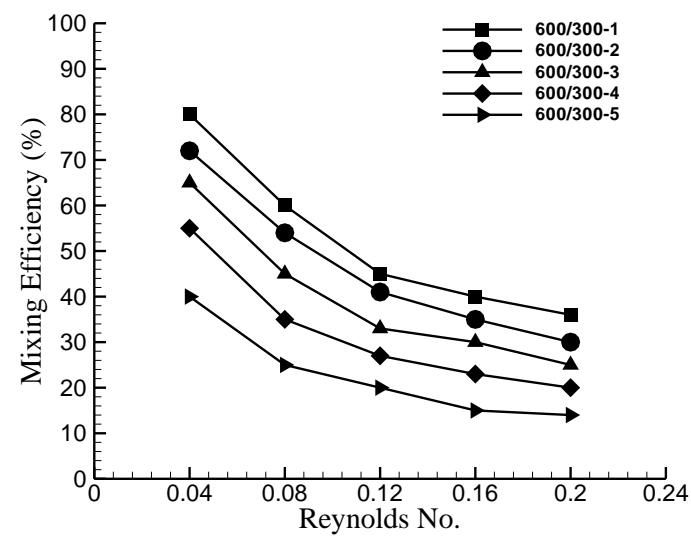

(c)

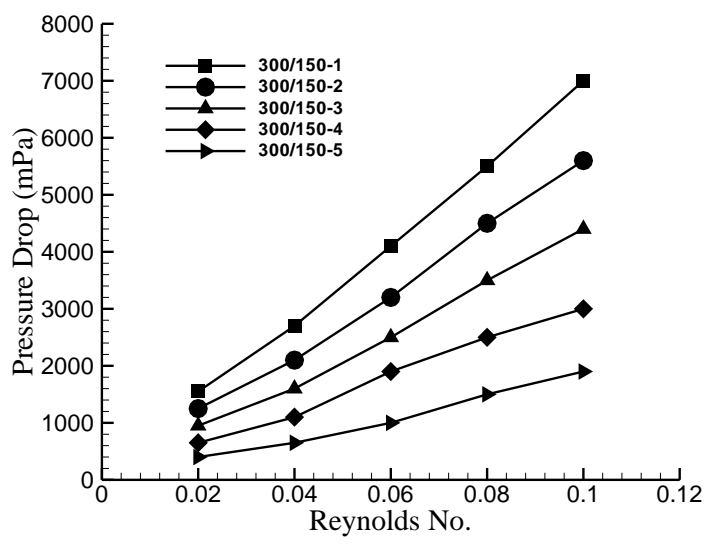

(b)

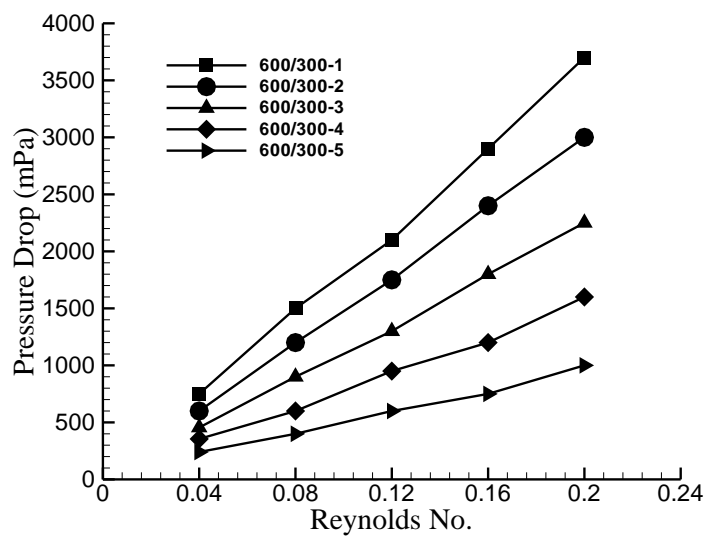

(d)

Fig. 2 Mixing efficiency and Pressure drops of the PDM-I and PDM-II geometries. (a) \& (c) depict the mixing efficiencies of the PDM-I and II geometries at various Re flows respectively. (b) \& (d) shows the pressure drops of the species calculated at outlet of the PDM-I and PDM-II geometries respectively for various Re flows. The legends in the Figs denote the width/height of the PDM geometries with corresponding number of dumbbell units. 


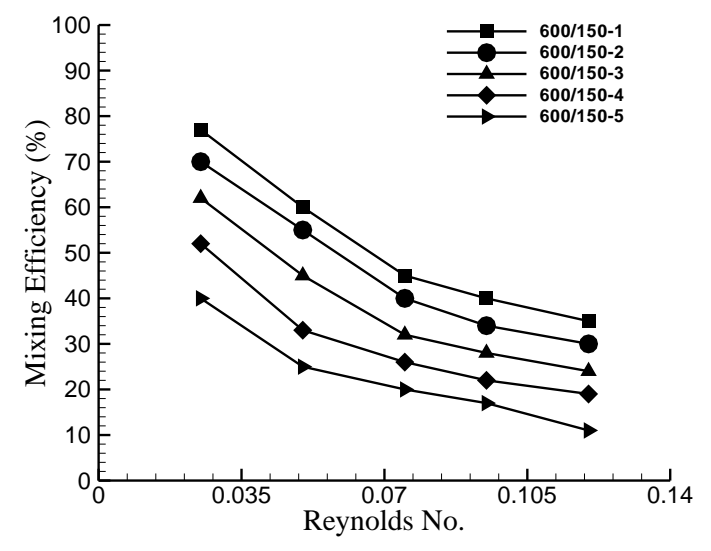

(a)

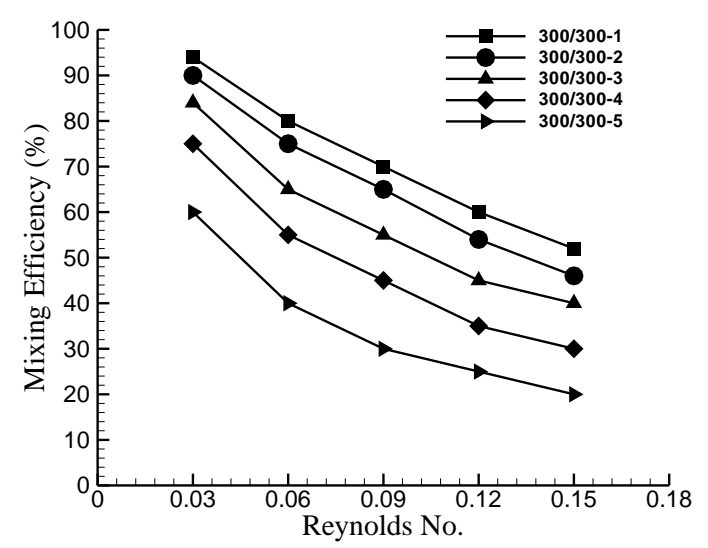

(c)

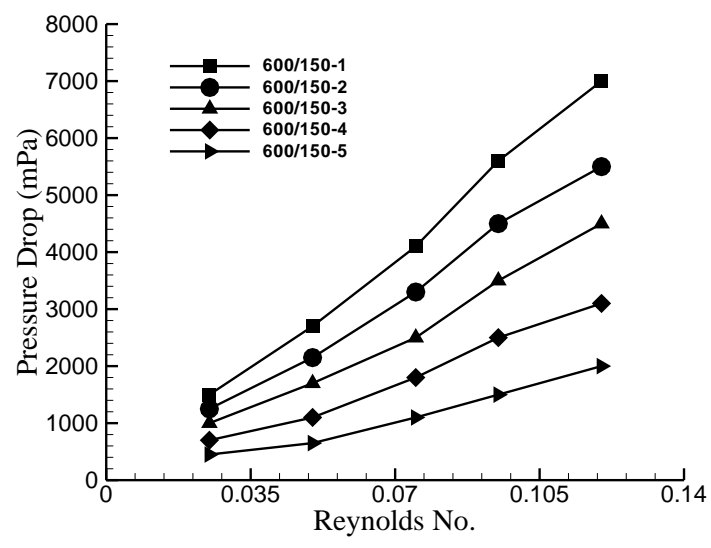

(b)

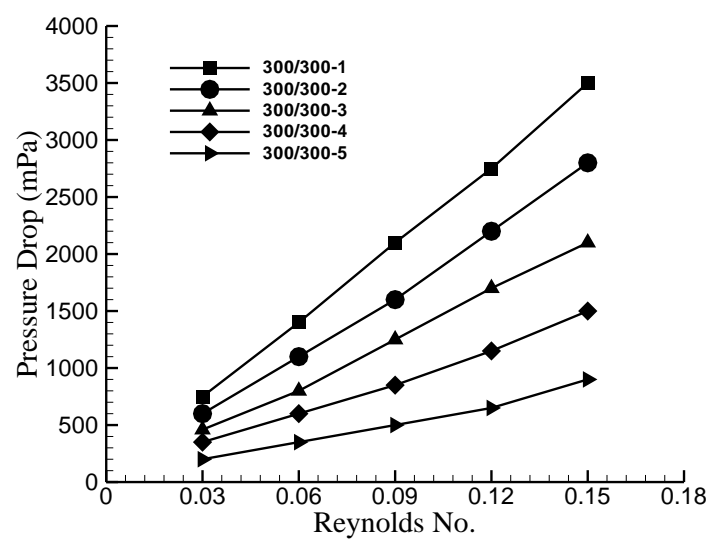

(d)

Fig. 3 Mixing efficiencies and Pressure drops of the PDM-III \&PDM-IV geometries. (a) \& (c) depict the mixing efficiencies of the PDM-III and PDM-IV geometries at various Re flows respectively. (c) \& (d) shows the pressure drops of the species calculated at outlet of the PDM III and PDM IV geometries respectively for various Re flows. The legends in the Figs denote

the width/height of the PDM geometries with corresponding number of dumbbell units.

$$
\Delta P=\frac{4 L \tau_{w}}{D_{h}}
$$

Where $\mathrm{L}$ is the length of the geometry. Increase in the height (for the case of PDM-IV geometry) does not affect the transverse diffusion of the species. The length $(\mathrm{L})$ to height $(\mathrm{H})$ ratio and the Peclet number (Pe) of the PDM I and IV fall in the regime of, $L /_{H} \gg P e \gg 1 \mathrm{In}$ this regime, the transverse diffusive transport is independent of the non-uniform velocities that arise due to increase or decrease of the height of the micromixer geometry, which has been experimentally corroborated [18]. 


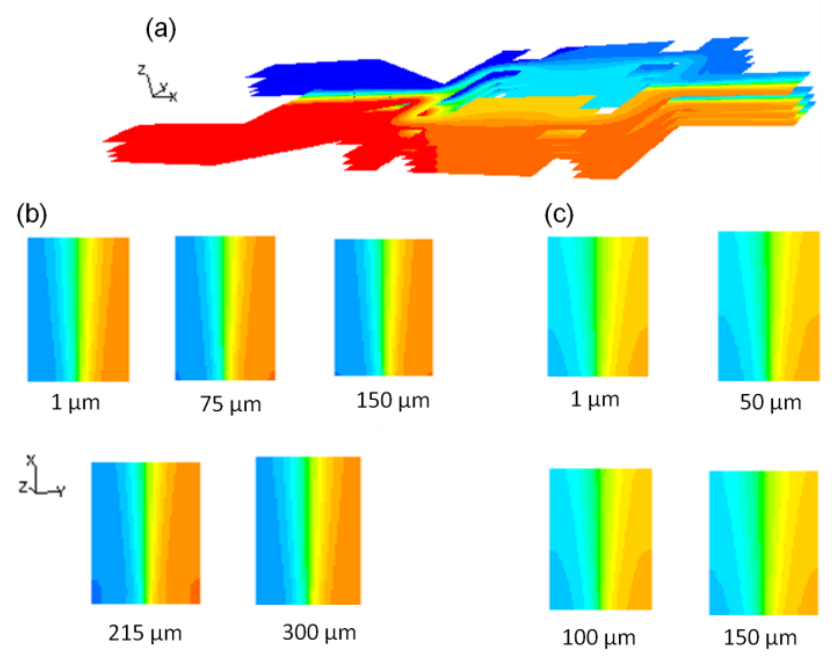

Fig. 4(a) The planes represent the Mass fraction of the water species at Re 0.02 at different height (z) of the PDM-II of the geometry; (b)\&(c) demonstrate the longitudinal diffusion broadening at the outlet plane at different height of the PDM-II and PDM-I at the velocity of $100 \mu \mathrm{m} / \mathrm{sec}$ of the water species respectively.

In order to comprehend the deviation (mixing efficiencies of species) from the scaling effect in PDM-II, the contours of the mass fraction of species at different height (planes) of the outlet channel of the PDM-I and PDM-II geometries are shown in Fig 4. The contours of mass fraction of species at the outlet top plane $(150 \mu \mathrm{m})$ and the bottom plane $(1 \mu \mathrm{m})$ of PDMI (Fig 4c) depicts the diffusion broadening and the diffusion is narrow at the geometry centre, which is termed as "butterfly effect" [23]. In the case of PDM-II, the diffusion broadening is narrow at the centre, while it is more than the half of the width of the channel at bottom and top planes shown in Fig 4(b). This is why the mixing efficiencies of the PDM-II does not fall half to that of the mixing efficiencies of PDM-I. The diffusion broadening at top and bottom surfaces across the width of the channel is generally high, since the flow velocity is minimum [18]. This explicates the characteristic of the diffusion phenomenon not adhering to the scaling effect, but on the velocity of the species across the geometry. The Fig 4(a) shows the contours of mass fraction of water species at different height $(z)$ of the PDM-II geometry with one dumbbell unit.

For the case of geometrically similar PDM-I and PDM-II geometries, dimensional analysis was performed pertaining to mixing efficiencies and pressure drops of the geometries. The dependence of a quantity and its relation with the variables can be sorted feasibly with the well-known dimensional analysis [25]. The pressure drop of a dynamic fluid in the geometry depends on the dimensions of the geometry and the properties of the fluid. Dimensional analysis gives a relation between the pressure drop and its dependent variables, given by [25]

$$
\frac{\Delta P}{\rho V^{2}}=f\left(\frac{H}{L}, \frac{\rho V D}{\mu}\right)
$$

Where $\Delta P$ is the pressure drop, $H$ is the height of the inlet channel/geometry, $L$ is the length of the geometry, $D$ is the diameter of the channel, $\rho$ is the density of the fluid, $V$ is the flow velocity of the fluid in the geometry and $\mu$ is the kinematic viscosity of the fluid.

The PDM-I and PDM-II are geometrically similar, in which their dimensions differ by a constant scale factor of two. Both PDM-I and PDM-II being similitude and using the relation Eq.(7) the pressure drops of both the geometries are related by

$$
\Delta P_{I}=2 \Delta P_{I I}
$$


Another parameter of interest here is the mixing efficiency of the fluids in the geometries. The mixing efficiency $(\eta)$ possibly depends on the variables like velocity $(V)$ of the fluid in the geometry, diffusion coefficient $(D)$ of the fluid, width/diameter $(W)$ of the geometry and the length $(L)$ of the geometry. Dimensional analysis on the mixing efficiency reveals the relation with its variables given by,

$$
\eta=f\left(\frac{D}{V L}, \frac{L}{W}\right)
$$

On the same lines as that of the pressure drop, the mixing efficiency of the fluids in the geometrically similar PDM-I and II geometries are related by

$$
\eta_{I I}=\frac{1}{2} \eta_{I}
$$

The Eq's (8) and (10) result from invoking the Buckingham PI theorem in the formulation of dimensional analysis. The Eq. (8) predicts, that the pressure drops of the PDM-I geometry to be twice that of the PDM-II geometry. Since, the mixing efficiency represents the percentage of mixing of fluids, from the Eq. (10) it is deciphered that the mixing efficiencies of species PDM-II are to be $50 \%$ of PDM-I. The relations given in Eq's (8) and (10) by dimensional analysis, reciprocate the same scaling effects phenomenon for the geometrically similar PDM-I and PDM-II geometries.

The simulation results of the geometrically similar PDM-I and PDM-II geometries illustrate the violation of the scaling and dimensional laws in concern to the mixing efficiencies [Fig $2 \mathrm{a}$ and $2 \mathrm{c}$ ] of species. In this regard, it is required to note of the diffusion

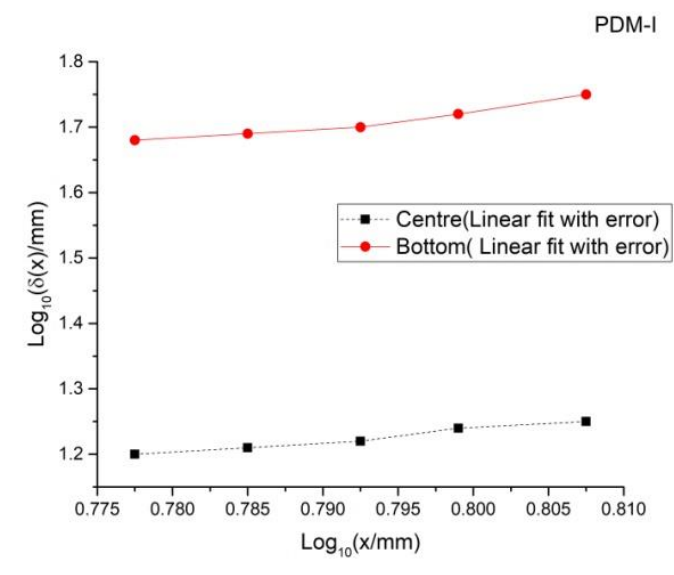

(a) PDM I

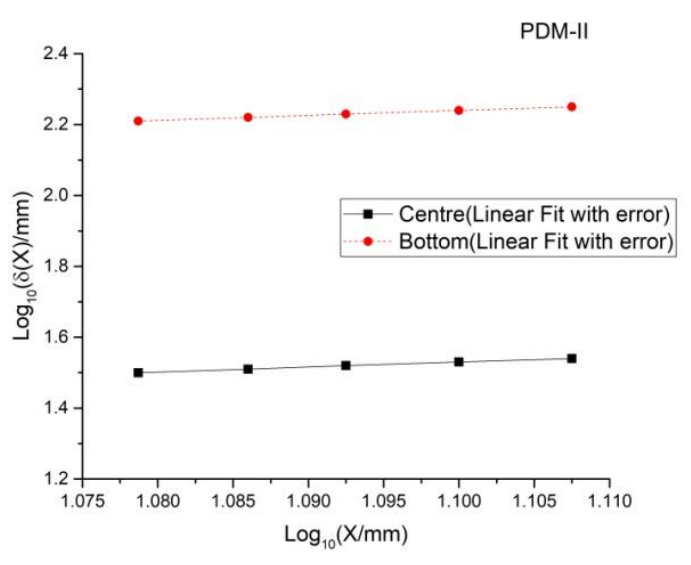

(b) PDM II

Fig. 5 The interfacial diffusion layer thickness $(\delta)$ as a function of the downstream axial distance x of (a) PDM-I and (b) PDM-II geometry; at the centre and bottom wall of the outlet channel. The lines drawn across the symbols represent the fitted lines.

Scaling law in passive micromixers, which was analyzed theoretically [26] and experimentally [19]. According to the law, the interfacial diffusion layer width is proportional to a power law of the ratio of the axial distance to the average flow velocity [23], which is mathematically represented by

$$
\delta \sim\left(\frac{x}{U_{\text {avg }}}\right)^{n}
$$

where, $\delta$ denotes the width of the interfacial diffusion layer, $\mathrm{x}$ is the axial distance and $U_{\text {avg }}$ is the average velocity of the species at the axial distance. Before embarking on the diffusion scaling law, the directionality of the diffusion of species in the rectangular PDM geometries is 
indeed necessary. In the fully developed flow in a microfluidic device, the species streamlines have non-uniform velocities across the geometry in the transverse direction. This makes the diffusion of species dominant in the transverse direction rather than depth-wise and axial diffusion. In the simulations of water species in the PDM (I,II,III and IV) geometries, the transverse diffusion overwhelmed the depth-wise diffusion, in which $W^{2} / D \gg H^{2} / D$, where $W$ and $H$ are the width and Height of the geometries respectively and $\mathrm{D}$ is the diffusion coefficient of the water species. But the depth-wise diffusion of species is more than the axial convection (along flow direction $\mathrm{X}$ ) of the species. Since $H^{2} / D \gg L / U_{\text {avg }}$ in all the PDM geometries, where $L$ is the length of the PDM geometry. Thus the diffusion broadening shown in Fig 4 at bottom walls of the channels is attributed to transverse diffusion.

The Fig 5 shows the width of the interfacial diffusion of species at the outlet channel wall and at the half-depth plane of the PDM-I and PDM-II geometries with respect to the axial distance x. In Fig 5a the scaling relationship between $\delta$ and the axial distance x of the PDM-I geometry is depicted. From the slope of the fitted line, the diffusion width $\delta$ scales as the $3 / 2$ power $(n=1.524$ deduced from the fitted line) of the axial distance $\mathrm{x}$ at the central plane and $9 / 5$ power ( $n=1.835$ deduced from the fitted line) at the bottom wall of the outlet channel. Similarly in the case of PDM-II shown in Fig 5b, the width of the interfacial diffusion layer $\delta$ scales as the $6 / 5$ power ( $n=1.260$ deduced from the fitted line) of the axial distance $\mathrm{x}$ at the central plane and $3 / 2$ power ( $n=1.479$ deduced from the fitted line) at the bottom wall of the outlet channel. From the Fig 5, the diffusion scaling law vary at bottom and central plane of the microchannel, further the diffusion scaling relations in the geometrically similar micromixers does not relate or show the scaling effect. Since the width of the diffusion layer at central and bottom walls of PDM-I and PDM-II outlet microchannels with respect to axial distance vary invariably.

\section{$4 \quad$ Validation of Dimensional Analysis}

In the validation of dimensional analysis, the simulation results of PDM-II geometry depicted in Figure 2 (c) and 2(d) were used. As shown in the Figure6, the predicted mixing efficiencies and pressure drops of PDM-II by dimensional analysis is compared with the calculated mixing efficiencies and pressure drops of the simulated PDM-II geometry. From the dimensional analysis the mixing efficiencies (equation 10), of the species in PDM-II were to be $50 \%$ higher to that of the PDM-I. This prediction falls gradually with addition of mixing units to the PDM-II geometry. As shown in the Figure5(a), the dimensionally predicted $50 \%$ is measured against the rise in percentage of mixing efficiency of PDM-II with respect to PDM-I mixing efficiency for one to five mixing units. The rise in percentage was calculated from the average of the percentage of five Re flows of the PDM-II mixing efficiencies with respect to PDM-I geometry. At low Re flows pure diffusion predominates and this should increase with increase in distance or length of the geometry, but the scaling factors limit the transverse diffusion as shown in the Figure4. The dimensional analysis prediction of mixing efficiency for the geometrically similar PDM-I and II was strictly valid up to two rectangular units of the geometry. Beyond which the error between predicted and actual values gradually increases with increase in number of sequential units.

In the case of pressure drops shown in Figure 6 (b), the dimensional analysis holds (equation 8), since the calculated average rise in percentage of pressure drop for five Reynolds Number ( $\mathrm{Re}$ ) flows is $\sim 90 \%$ and is consistent for one to five mixing units. The average rise in percentage of pressure drop was calculated from the simulations of individual units of PDM I and II. The non-uniform velocities, which arise by reduction of dimensions of the geometry, impose the consistent wall shear stresses, thereby the pressure drops in PDM-II maintain twice that of the PDM-I geometry for all the units. The $10 \%$ variation ofpressure 
drops in simulation and dimensional analysis may be attributed to volume cells mismatch between simulated PDM-I and II. Thus, the dimensional analysis predicts the pressure drops satisfactorily for the geometrically similar passive micromixers.
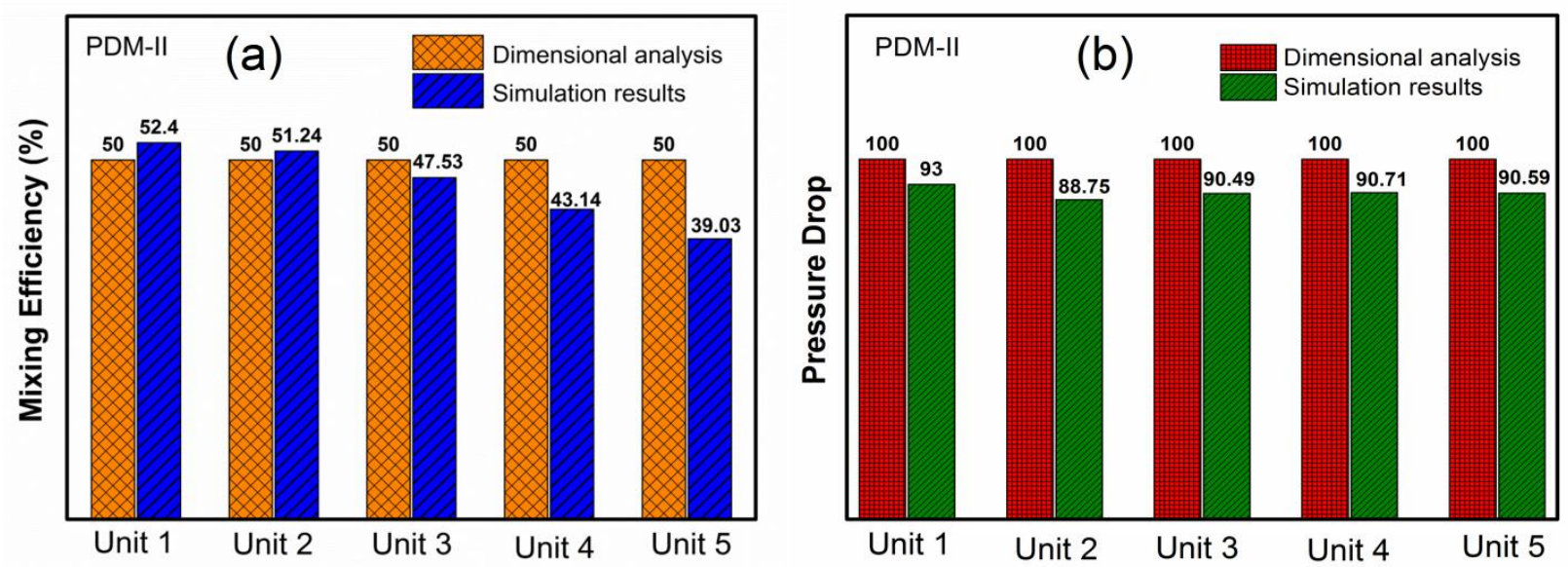

Fig. 6 Comparison of the (a) mixing efficiencies; and (b) pressure drops; of the PDM-II geometry with one to five rectangular dumbbell units.

In the complex LOC device or a micromixer, rigorous iterations are necessary to understand the functionality and the reproducibility of the device. In this regard, a model with bigger dimensions, i.e. with a constant $\mathrm{X}$ scale factor to that of the prototype can be constructed to predict the parameters of interest through dimensional analysis. The studies were done in the low Re regime to understand the scaling effects on the diffusion phenomenon. But, the dimensional analysis find its relevance to some extent after which it widens the gap between the predicted and the actual mixing efficiencies as shown in Figure 6(a). Further studies are required to introduce the correction factors in the dimensional analysis for the better prediction of all relevant parameters of interest in the prototype by working on the model. Also, studies can be done in the high Re regime i.e. 50 to 200, where the chaotic advection replaces diffusion in the micro geometries. The drawbacks of the simulations are the numerical diffusion, which imposes errors in the analysis. In this work, it was mitigated by structural meshing of all the simulated PDM geometries with only hexahedral cells. Moreover, the ratio of number of meshed cells in the geometrically similar PDM-I and II for one to five rectangular dumbbell units is same. The dimensional analysis is the feasible way for impossible or prohibitively expensive experiments, which makes use of scaling laws in the geometrically, kinematically and dynamically similar geometries or objects. The dimensional analysis of the microscale flows indeed predict the pressure drops accurately. Moreover, the present study demonstrates the novel rectangular dumbbell structure for efficient mixing of species in the passive micromixer geometries.

\section{Fabrication and validation of simulation studies of passive micromixers}

Introduction of polymers in the micro technologies is the best alternative to silicon and other inorganic materials for fabrication of micromixers and complex microdevices. Polydimethylsiloxane is one of the successful elastomeric materials widely employed in soft lithography, a microtechnology based on direct pattern transfer technique. In most of the fabricated microfluidic devices, the elastomeric PDMS was used with microchannels embedded in them. Briefly in this, the process starts with the fabrication of master with sophisticated techniques such as photolithography employing thick resist film of SU-8. Followed by this step, the PDMS mixture (pre-polymer base and curing agent in 10:1 ratio) is poured on to the master and cured at temperatures 60 to $80^{\circ} \mathrm{C}$ for $3-4 \mathrm{~h}$. Next, the PDMS with 
patterns can be seen after it is peeled off from the master. Now the patterned PDMS brought into contact with silica or glass or another flat PDMS by surface treating the both with oxygen plasma. Soft lithography is the economical method and a rapid prototype technique. The vast number of reported lab scale prototypes were fabricated with this technique.

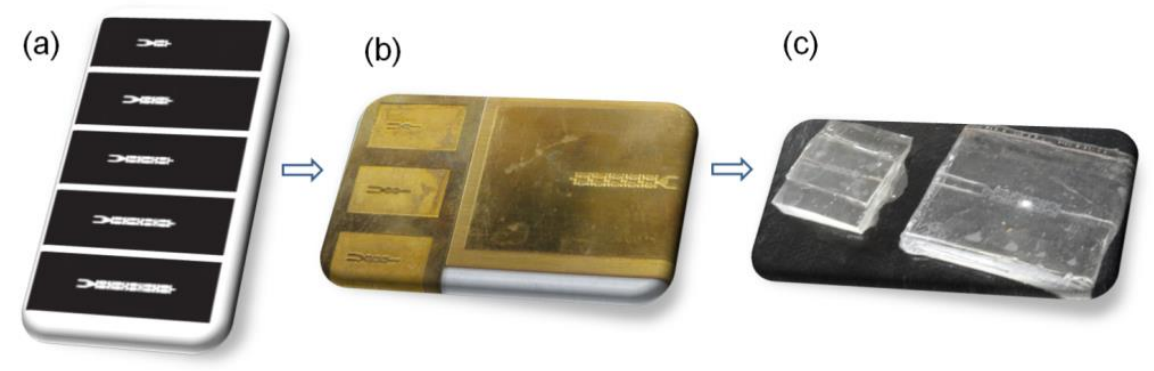

Fig. 7 Fabrication stages of the Passive dumbbell micromixer (a) positive photo mask developed on a transparent sheet with a high DPI printer (b) the designs transferred on brass plates by transfer toner and etching techniques (c) the designs on brass replicated on PDMS and bonded by PDMS-PDMS bonding

The passive micromixers reported in this work were fabricated by template based soft lithography method. Initially the novel designs (2D) of the passive micromixers PDM-II and III, with one to five rectangular dumbbell units were drawn in CorelDraw software. These designs were imprinted on a transparent sheet using a commercially 1200 DPI printer. Using the positive photo mask sheets and employing the transfer toner method and etching techniques the designs were replicated on the commercially obtained brass plates $(0.5 \mathrm{~mm}$ thickness) with the height of the geometries as $150 \mu \mathrm{m}$. Before using the brass plates, copper board with glass epoxy substrate was used. The problem with the copper boards was, commercially the maximum available copper thickness is less than $100 \mu \mathrm{m}$. Hence, we could not achieve $150 \mu \mathrm{m}$ height for the geometries after the etching of the patterned copper board in the ferric chloride solution. After obtaining the patterned micromixer designs on the brass plates, these were cleaned by ultrasonication for 15 minutes by immersing in methanol in a glass beaker. The cleaned brass plates were individually placed in the plastic boxes and PDMS (Sylgard 184, Dow corning) mixture (10:1; pre-polymer: base) was poured on the brass plates. After the selflevel of the PDMS in the box with no air bubbles trapped, the box was placed in an hot oven at $65^{\circ} \mathrm{C}$ for $3 \mathrm{~h}$. Next, the cured PDMS (5mm thickness) was carefully peeled off from the brass plates with rectangular passive micromixers patterned on one surface into the PDMS. The next task here was closing of the open rectangular structures on PDMS surface, we thought do it by PDMS-PDMS bonding. In this, a flat PDMS surface was bonded with the patterned PDMS. After several trials and constant monitoring, we could achieve PDMS-PDMS bonding withstanding high flow rates greater than $2 \mathrm{ml} / \mathrm{min}$. In this, a $1 \mathrm{~mm}$ thick PDMS was semi-cured, at an optimized temperature and time of $70^{\circ} \mathrm{C}$ and 45 minutes respectively. Over this, the patterned PDMS was placed and gently applied the pressure force from top on all sides and cured the PDMS layer structures for $2 \mathrm{~h}$ at $65^{\circ} \mathrm{C}$. Having some practical difficulties and reverse flow in the fabricated micromixer geometries, the inlet channel lengths was increased to $5 \mathrm{~mm}$. The empirically developed PDMS-PDMS bonding technique was very economical and very productive which can withstand flow rates more than $2 \mathrm{ml} / \mathrm{min}$.

From the simulation results, discussed in section 3, PDM-II, which is geometrically similar to that of the PDM-I comply with the dimensional analysis in terms of the pressure drop and partially comply to some extent in mixing efficiencies. The Figures 2(b) and (d) illustrate the 
pressure drop values of PDM-II are twice that of the PDM-I. The mixing efficiencies of PDM-II increase by $50 \%$ to $39 \%$ for one and five dumbbell units respectively, with respect to PDM-I geometry, as shown in Figure 2(a) and (c). While, the simulations of the PDM-III geometry demonstrate the mixing efficiency and pressure drop of the species, shown in Figure 3(a) and (b) respectively. In this, the height of the PDM-III geometry half to that of the PDMI resulted in no change in mixing efficiency and the pressure drops increased thrice. In this purview, the PDM-II and PDM-III are optimally adequate and inadequate respectively. Hence, these two geometries are chosen for fabrication and for validation with the simulation results in terms of the mixing efficiency of the species and liquid dyes.

The PDM-II and III micromixer geometries have been fabricated in PDMS by the method described just above this subsection. The micromixers are PDMS-PDMS bonded with four rectangular dumbbell mixing units chosen in which the liquid dyes were mixed to validate the simulation results.

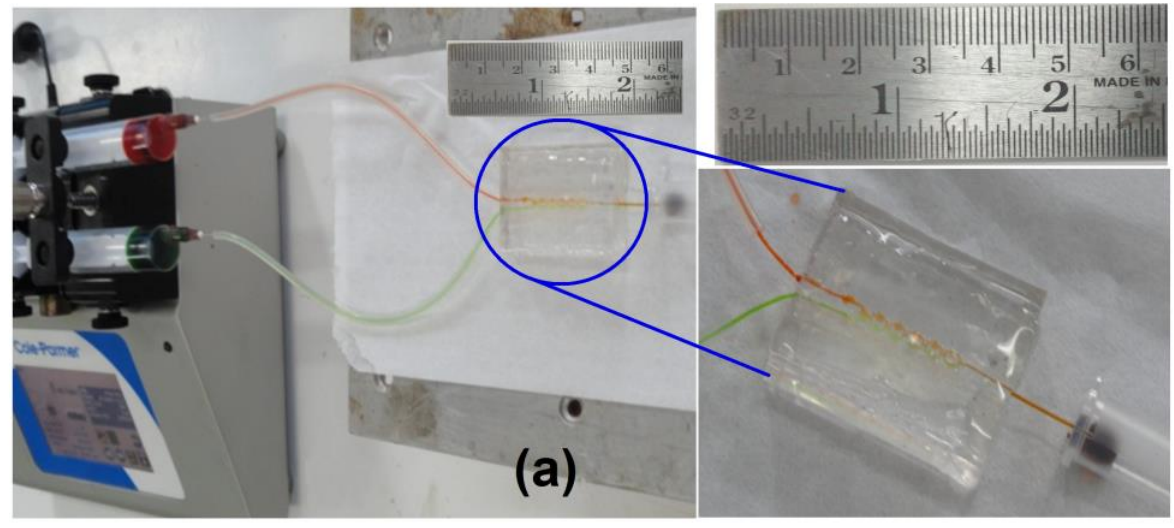

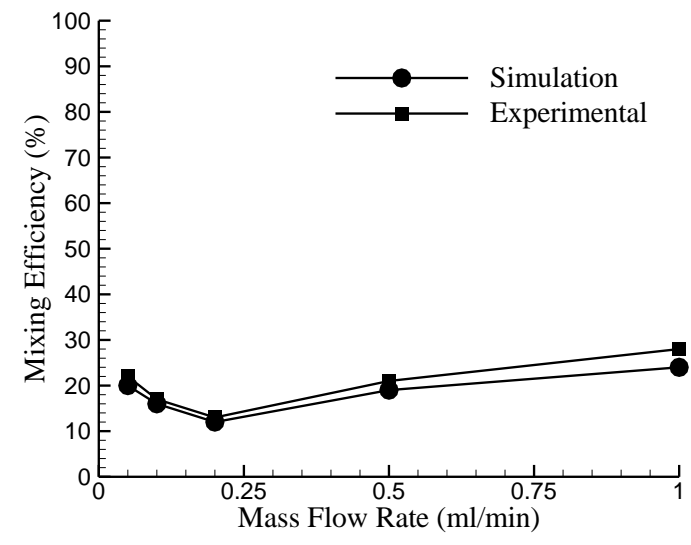

(b)

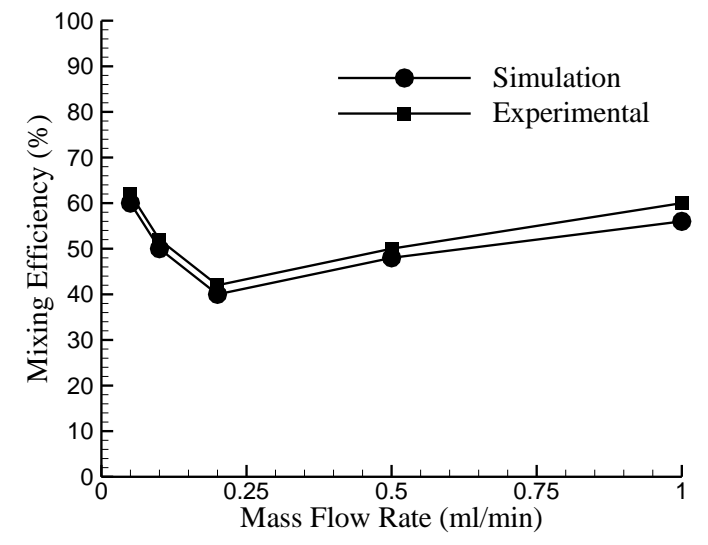

(c)

Fig 8 (a) Experimental setup of the dyes mixing in the PDM-III geometry with five dumbbell structures. The PDM-III inlets connected to the syringes (mounted on syringe pump) through the silicone tubes. Inset shows the exploded view of Fabricated PDM-III. (b) The simulated and experimental mixing efficiencies of the PDM-III geometry for various mass flow rates of the water species and dyes respectively. Similarly, (c) The simulation and experimental mixing efficiencies for the PDM II geometry; with four mixing units in the geometry

The orange and green food dyes of same quantity (1gram) were dissolved in $20 \mathrm{~mL}$ of water individually. It was made sure that there are no fine granules of colour pigments in the solution, which could deposit in the syringe needle or in the microchannel and block the flow. The quantity of 1 gram of food dyes in $20 \mathrm{~mL}$ of water was good enough, which give bright colors of orange and green, to flow through the transparent PDMS channels. The syringefilled dyes were mounted on the syringe pump (Make: Cole Parmer). The inlets of the 
fabricated PDMS PDM-II micromixer with four sequential mixing units, connected with the silicone tubes to the syringes as shown in the experimental setup in Figure8(a). The mass flow rates of the dyes into the PDM micromixer were controlled through the syringe pump from $0.05 \mathrm{ml} / \mathrm{min}$ to $2 \mathrm{ml} / \mathrm{min}$. Images were recorded with Canon EOS 550D focusing on the outlet channel of the micromixer at every flow rate of the dyes into the micromixer. Similarly, in the PDM-III the same setup as described for PDM-II micromixer was followed for dyes mixing and the images at the outlet were recorded at every flow rate considered. Next, Image analysis of the dyes mixing at the outlet of the PDM-II and III was done by Image $\mathbf{J}$ software. The Figure8(b) and 8(c) shows the mixing efficiencies of the dyes calculated at different mass flow rates in the PDM-II and III geometries respectively and compared with the simulation results of species mixing at the same flow rates. For the case of PDM-II geometry in the Figure8(b), the experimental and simulation, mixing efficiencies were in good agreement at every mass flow rates. Here, the variation is $5-10 \%$ at low mass flow rates and 15 to $20 \%$ at higher mass flow rates. This may be attributed to the roughness of the fabricated channels. From the analysis in simulations for PDM-III geometry, the top and bottom wall roughness may enhance the transverse diffusion and advection along the depth of the channel. This phenomenon induces more mixing of dyes in the fabricated micromixers, where as in simulations, no slip condition was enforced at the walls of the geometries. In the case of Figure8(c), the simulation and experimental mixing efficiencies are in good agreement (with variation of 2-3\%) at low mass flow rates only, at which diffusion phenomenon is prevailed. At higher mass flow rates discrepancies are observed, again due to the roughness of the walls of the fabricated PDM-III channels, which can induce strong chaotic advection of the dyes in the geometry.

\section{CONCLUSION}

The study was aimed at understanding the scaling effect on mixing efficiencies and pressure drops in the geometrically similar PDM-I and II passive micromixers. Extensive simulations of the PDM geometries in the Ansys Fluent show the pressure drops of PDM-I was twice that of the PDM-II, while the mixing efficiencies of PDM-II are not half to that of PDM-I. The simulations of PDM-III and IV geometries which vary linearly in $\mathrm{Y}$ and $\mathrm{Z}$ directions to that of the PDM-I respectively, reveal that increasing the width decreases the mixing efficiencies, but not according to scaling effects. The pressure drops reduces to half with increase in depth of the channel twice, which are according to scaling effect and dimensional analysis. The diffusion broadening at the top and bottom walls of PDM-II was twice that of the channel width, this made the mixing efficiencies increase more than $50 \%$ of PDM-I. The velocities of the species streamlines are uniform except at the walls of the PDMII geometry, where the transverse diffusion increases, while it is same and minimum across the geometry. The PDM-I and III geometries were fabricated in PDMS by soft lithography. In these, the mixing efficiencies evaluated by dyes mixing, which demonstrate the scaling effect deviation on mixing efficiencies of dyes as that of simulation results of species mixing.

\section{REFERENCES}

[1] Ohno, K.I., Tachikawa, K., Manz, A. "Microfluidics: applications for analytical purposes in chemistry and biochemistry”, Electrophoresis 29, pp. 4443 - 4453, 2008.

[2] Zhang, C., Xu, J., Ma, W., Zheng, W. "PCR microfluidic devices for DNA amplification", Biotechnology Advances, 24, pp. 243 - 284, 2006.

[3] Elvira, K.S., Solvas, X.C., Wootton, R.C. "The past, present and potential for microfluidic reactor technology in chemical synthesis", Nature Chemistry 5, pp. $905-$ 915, 2013. 
[4] Demello, A. J. "Control and detection of chemical reactions in microfluidic systems". Nature 442, pp. 394 - 402, 2006.

[5] Andersson, H., Van den Berg, A. "Microfluidic devices for cellomics: a review", Sensors and Actuators B: Chemical 92, pp. 315 - 325, 2003.

[6] Becker, H., Gärtner, C. "Polymer microfabrication technologies for microfluidic systems", Analytical and Bioanalytical Chemistry 390, pp. 89 - 111, 2008.

[7] Morin, S.A., Shevchenko, Y., Lessing, J., Kwok, S.W., Shepherd, R.F., Stokes, A.A., Whitesides, G.M. "Using "Click-e-Bricks" to make 3D elastomeric structures", Advanced Materials 26, pp. 5991 - 5999, 2014.

[8] Qin, D., Xia, Y., Whitesides, G.M. "Soft lithography for micro-and nanoscale patterning" Nature Protocols 5, pp. 491 - 502, 2010.

[9] Becker, H., Locascio, L.E. "Polymer microfluidic devices". Talanta 56, pp. 267- 287, 2002.

[10] Lisowski, P., Zarzycki, P.K. "Microfluidic paper-based analytical devices ( $\mu$ PADs) and micro total analysis systems ( $\mu \mathrm{TAS})$ : development, applications and future trends", Chromatographia 76, pp. 1201 - 1214, 2013.

[11] Lee, S.W., Kim, D.S., Lee, S.S., Kwon, T.H. "A split and recombination micromixer fabricated in a PDMS three-dimensional structure", Journal of Micromechanics and Microengineering 16, 1067, 2006.

[12] Afzal, A., Kim, K.Y. "Passive split and recombination micromixer with convergentdivergent walls", Chemical Engineering Journal 203, pp. 182 - 192, 2012.

[13] Lee, J., Kwon, S. "Mixing efficiency of a multilaminationmicromixer with consecutive recirculation zones", Chemical Engineering Science 64, pp. 1223 - 1231, 2009.

[14] Nichols, K.P., Ferullo, J.R., Baeumner, A. J. "Recirculating passive micromixer with a novel sawtooth structure", Lab on a Chip 6, pp. 242 - 246, 2006.

[15] Tofteberg, T., Skolimowski, M., Andreassen, E., Geschke, O. "A novel passive micromixer: lamination in a planar channel system", Microfluidics and Nanofluidics 8, pp. $209-215,2010$.

[16] Hessel, V., Löwe, H., Schönfeld, F. "Micromixers - a review on passive and active mixing principles”. Chemical Engineering Science 60, pp. 2479 - 2501, 2005.

[17] Veldurthi, N., Chandel, S., Bhave, T., Bodas, B. "Computational fluid dynamic analysis of poly(dimethyl siloxane) magnetic actuator based micromixer", Sensors and Actuators B: Chemical 212, pp. 419 - 424, 2015. DOI: 10.1016/j.snb.2015.02.048

[18] Kamholz, A.E., Weigl, B.H., Finlayson, B.A., Yager, P. "Quantitative analysis of molecular interaction in a microfluidic channel: the T-sensor", Analytical Chemistry 71, pp. 5340 - 5347, 1999.

[19] Ismagilov, R. F., Stroock, A. D., Kenis, P. J., Whitesides, G., Stone, H. A. "Experimental and theoretical scaling laws for transverse diffusive broadening in twophase laminar flows in microchannels", Applied Physics Letters 76, pp. 2376 - 2378, 2000.

[20] Sullivan, S. P., Akpa, B. S., Matthews, S. M., Fisher, A. C., Gladden, L.F., Johns, M. L. "Simulation of miscible diffusive mixing in microchannels", Sensors and Actuators B: Chemical 123, pp. $1142-1152,2000$. 
[21] Chen, J. M., Horng, T. L., Tan, W. Y. "Analysis and measurements of mixing in pressure-driven microchannel flow", Microfluidics and Nanofluidics 2, pp. $455-469$, 2006.

[22] Morf, W. E., Van der Wal, P. D., De Rooij, N. F. "Computer simulation and theory of the diffusion-and flow-induced concentration dispersion in microfluidic devices and HPLC systems based on rectangular microchannels", Analyticachimicaacta 622, pp. 175 $-181,2008$.

[23] Song, H., Wang, Y., Pant, K. "Scaling law for cross-stream diffusion in microchannels under combined electroosmotic and pressure driven flow", Microfluidics and nanofluidics 14, pp. 371 - 382, 2013.

[24] Engler, M., Kockmann, N., Kiefer, T., Woias, P. "Numerical and experimental investigations on liquid mixing in static micromixers", Chem. Eng. J. 101, pp. $315-$ 322, 2004.

[25] Fox, R. W., McDonald, A. T. "Introduction to Fluid Mechanics", 5 ${ }^{\text {th }}$ Edition, Wiley, 2001.

[26] Ayodele, SG., Varnik, F., Raabe, D. "Effect of aspect ratio on transverse diffusive broadening: a lattice Boltzmann study", Phys Rev E 80(1):016304, 2009.

[27] Stefan, G., Dzianik, F., Martin, J., Kabat, J. "Shell and Tube Heat Exchanger - the Heat Transfer Area Design Process", Journal of Mechanical Engineering - Strojnícky časopis 67 (2), pp. 13 - 24, 2017. DOI: 10.1515/scjme-2017-0014

[28] Chribik, A., Poloni, M., Lach, J., Jancosek, L., Peter, K. Zbranek, J. "Internal Combustion Engine Powered by Synthesis Gas from Pyrolysed Plastics", Journal of Mechanical Engineering - Strojnícky časopis 66 (1), pp. 37 - 46, 2016. DOI: 10.1515/scjme-2016-0009 